\title{
Deployment Forecasts of China's Photovoltaic: Based on Multi- model Comparison Analysis
}

\author{
Fang Yang ${ }^{1}$, Xiaoliang Tang ${ }^{1}$, Weicong Ruan ${ }^{1}$ \\ Qingyuan Power Supply Bureau of Guangdong Power Grid Company, 511000 Qingyuan, Guangdong
}

\begin{abstract}
Climate targets in Paris Agreement closely depends on the deployment of renewables, particular for solar photovoltaic (PV) technology, which also plays formidable role in achieving China's Intended Nationally Determined Contributions (INDC) goals in 2030. On this basis, we conduct a multi-model comparison study to examine the diffusion laws of PV solar technology across countries, and provide feasible deployment forecasting plans for China. The considered models in this work includes classical Bass, Logistic and Gompertz; and 10 countries with top PV cumulative capacity are involved, i.e., France, Germany, Italy, Spain, the UK, Australia, China, India, Japan and the US. Our results depict that China gains remarkable advantage in PV solar technology innovation; the PV market has a great potential to grow, with the projected annual growth rate to be $30 \sim 40 \%$ annually in the coming 5 years. Further, several policy recommendations are yielded for China's long-term development of PV solar industry.
\end{abstract}

\section{Background}

The increasingly severe global warming and local air and air fog and haze have produced an urgent demand for clean electricity, which provides a good opportunity for the development of renewable energy technology such as photovoltaic solar energy. Based on this, China has promised to achieve the peak of carbon emissions by 2030 in the Paris agreement and the dual target of increasing the proportion of non fossil energy to the primary energy demand (TPED) to about $20 \%$. Obviously, the final realization of these goals is inseparable from the scale development of photovoltaic technology.

The natural resources of solar radiation in China are very good. The radiation resources of the Qinghai Tibet Plateau, the northern Gansu, the east of Xinjiang and the central and Western Inner Mongolia are particularly abundant, and the annual total radiation of the Qinghai Tibet Plateau is up to $1800 \mathrm{kw} * \mathrm{~h} / \mathrm{m}^{2}$ ([1-2]). In recent years, under the severe international greenhouse gas emission reduction situation and the dual incentive of domestic energy structure adjustment, China's photovoltaic industry has been unprecedented development. Since 2015, China has a total of 43.53 billion watts of cumulative installed capacity in the world, by 2016, this value has increased to 78.7 billion watts, and the cumulative increase in the past 5 years has been nearly 20 times ([3]).

The potential of solar energy market development in our country is expected to rise steadily in the future. With the great potential of solar energy resources and the signal of good policy. However, the trend of policy and the accurate prediction of the potential of market development are still the key factors affecting the development path of medium and long term technology ([4]).

Compared with other countries, what is the law of the diffusion path of photovoltaic power technology in our country, what is the technological innovation ability of the solar industry in our country, what is the pace of its future growth and the way to spread it, and how to set up an effective policy system so as to ensure the medium and long-term sustainable health of solar photovoltaic technology in China. These problems are related to the strategic choice of sustainable development of solar energy industry in China, the cultivation of photovoltaic power technology innovation ability, and the orderly promotion of the clean transformation of energy supply ([5]). In view of this situation, this paper has carried on the trans regional comparison, the analysis, the forecast.

\section{Model and method}

This paper first makes use of the parameter estimation of standard Bass model to compare the technical innovation ([6]) with imitation capability of the major power countries in the world. Secondly, it compares and analyses the medium and short term pretesting of China's extensive power technology diffusion through the Bass model (BM), the Gompertz model (GM) and the Logistic model (LM).

\subsection{Standard Bass model}

Captions should be typed in 9-point Times. They should be centred above the tables and flush left beneath the 
figures. Since its development in 1969, the Bass model has become a standard model to study the diffusion of product and technology innovation ([7-8]). Definitions in similar literature ([9]). Given $f(t)$ as the probability of using a technical product(this paper refers to solar photovoltaic technology) at $t$ time. $F(t)=\int f(t) d t$ is the cumulative probability of adoption at the end of $t$ time. The Bass model can be expressed as:

$$
f(t)=(1-F(t))(p+q F(t))
$$

It is assumed that the market potential of technology is $\mathrm{m}$, and the number of technology used at $t$ time is $k$. Then the cumulative amount of $K$ is $K(t)=\int k(t) d t$ at $\mathrm{t}$ time. Based on this, the upper form can be transformed into:

$$
\frac{d K(t)}{d t}=(m-K(t))\left(p+\frac{q}{m} K(t)\right)
$$

Among them, $p$ is the innovation coefficient of technological diffusion process, and $q$ is the imitation coefficient, representing the innovation ability and imitation level of technological development respectively. Generally speaking, the cumulative diffusion curve of the Bass model is approximately $\mathrm{S}$ type, while the specific line curvature depends on the parameters $p$ and $q$. For the high innovation technology $(p>q)$, the model will present a convex curve similar to exponential growth, that is, the diffusion rate is maximum at the beginning, and then decreases. On the contrary, for the highly imitated technology innovation $(p \ll q)$, the technology diffusion path presents a classic "S type". Therefore, the technology diffusion path under the Bass model can only be in between them. Generally speaking, the technological or product innovation diffusion processes belong to second types, that is, the imitative coefficient is far higher than the innovation coefficient. This also explains why the innovative diffusion curves of real observation are mostly "S" model or approximate "S" model. Especially for the Bass model, we can get the closed form solution of the system (2).

$$
K(t)=m \frac{1-e^{-(p+q) t}}{\left(1+\frac{q}{p} e^{-(p+q) t}\right)}
$$

\subsection{Logistic model}

The Logistic model originated from population prediction. Griliches ([10]) first used this model to discuss the adoption of American Hybrid Maize innovation, and then it developed into a representative model for the study of innovation diffusion ([11-12]).
The parameter $r$ is defined as the intrinsic growth rate of new technology or products. $K$ and $m$ denote cumulative usage and maximum potential respectively. Logistic model can be expressed as:

$$
\frac{d K(t)}{d t}=r K\left(1-\frac{K}{m}\right)
$$

The closed form solution of first order differential equation system (4) is obtained from the theory of ordinary differential equations.

$$
K(t)=K \frac{1}{1+c e^{-r t}}
$$

Actually, through variable displacement, logistic model (4) can be deformed as:

$$
\frac{\frac{d K(t)}{d t}}{m-K}=r \frac{K}{m}
$$

It is pointed out that the potential adopter number ( $m$ $K$ ) is positively related to the adopter $K$ multiplied by the coefficient $\mathrm{r} / \mathrm{m}$. If the decision of the potential adopter is also positively related to the effect value of the adopter, the system (6) can be understood as the network externality, that is, the utility of a certain adopter will increase with the increase of the technical adoption of other relevant adopters. There is also a scale effect on the demand side ([12]).

\subsection{Gompertz model}

The idea of the Gompertz model was first proposed by the British life insurance expert B. Gompertz in 1820 to predict population growth, and the American scholar R. Prescott was first applied to market prediction in 1922. Then, Chow ([13]) uses the model to analyze American computer needs and compares the predicted results with the standard Logistic model, providing an empirical evidence for the application of the Gompertz model in the field of technology diffusion ([14]).

Specifically, giving the definition of the intrinsic growth rate of product or technological innovation $r$. The definition of $K$ and $M$ is the same as above, the Gompertz model can be characterized by the following systems:

$$
\frac{d K(t)}{d t}=r K \ln \frac{m}{K}
$$

Since the equation (7) is a separable first order differential equation, the analytic solution is obtained:

$$
K(t)=m e^{-c e^{-r t}}
$$

Among them, $c=\ln \left(m / K_{0}\right), K_{0}$ is the amount of use of technology in the initial period. 


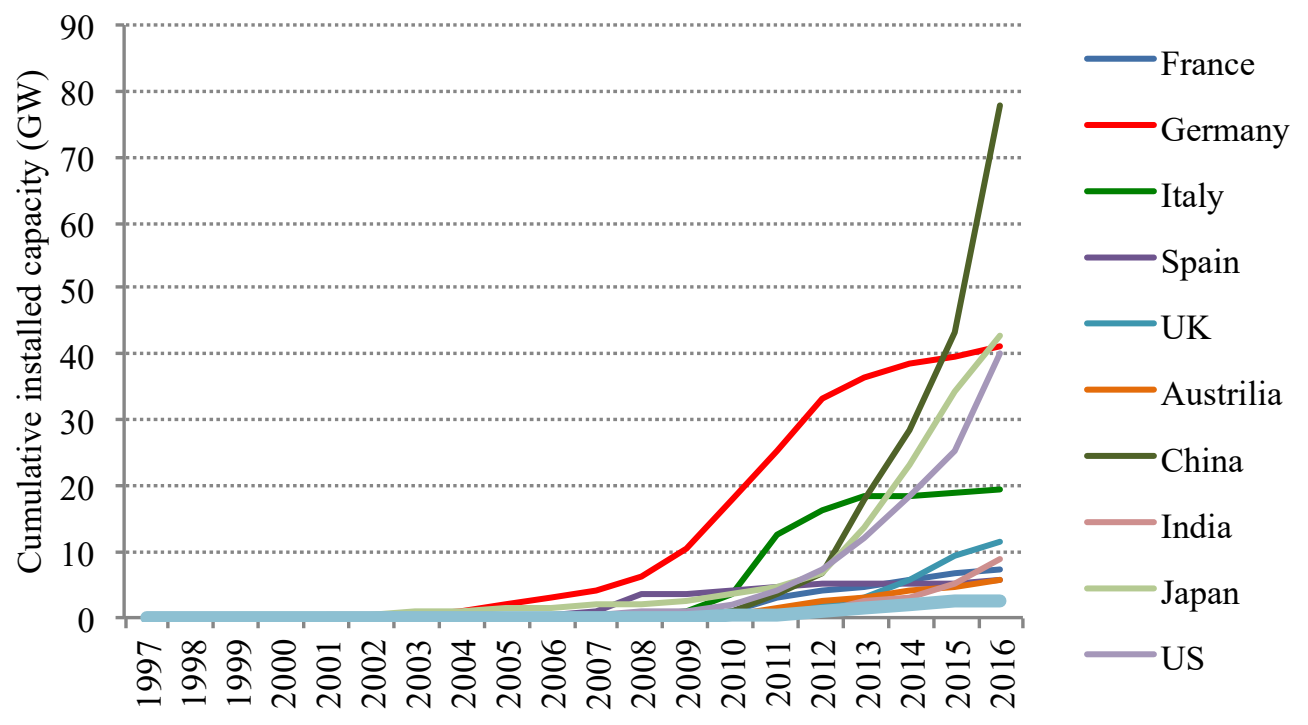

Fig. 1. Photovoltaic power installed capacity of major solar energy developing countries in the world

\section{Data and parameter estimation}

To analyze the overall development level of photovoltaic technology in China, This paper aims to compare the main solar power development capacity of the world's solar photovoltaic power installed capacity (annual installed capacity and cumulative installed capacity). Data came from 《BP world energy statistics 2016》 of BP. Data description as shown in picture 1 .

Model estimation is one of the most important parts of model application., For Bass model, the most commonly used method is least squares (OLS), This method is simple and easy to use, but it is prone to generate wrong signals and the stability of estimation results is also poor ([16]). Maximum likelihood estimation (MLE) is superior to OLS in terms of accuracy and stability, But there is a problem that underestimates the standard deviation. The estimation results of nonlinear least squares (NLS) are better than those of the former two, Therefore, it has become the main valuation technology adopted in this paper. To facilitate parameter estimation, we first differentiate the Bass model (2),

$$
\Delta K(t)=p(m-K(t))+\frac{q}{m} K(t)(m-K(t))
$$

The formulation $\Delta K(t)=K(t+1)-K(t)$ is New loader capacity 。

\section{4 empirical analysis}

\subsection{Transnational analysis of photovoltaic technology diffusion}

The comparison of the innovation and imitation level of $\mathrm{PV}$ power technology diffusion in different countries is shown in Table 1. As a whole, the imitating coefficient is greater than the innovation coefficient of Bass model, this shows that the innovation level of PV power technology is far below the level of imitation, no matter for the leading technology countries in Europe and America, or the lagging Asian countries. Therefore, the development of global solar technology is analogous to other technologies such as wind power. From a national point of view, Germany and the United States are the country with the highest level of photovoltaic technology innovation, in fact, the two countries are also the ranks of the countries with the highest accumulative installed capacity, Its installed capacity in 2016 was 41275 and $40300 \mathrm{MW}$, Obviously, As a pioneer of the development of photovoltaic solar technology, the two countries have accumulated rich experience in technological development over the past 20 years, providing a solid foundation for the in-depth innovation and large-scale imitation of photovoltaic technology. Although the largescale development phase of China's solar photovoltaic technology began late, it was not until 2010 that it was among the top 10 in the world, but the advantages of China's technology are very obvious, From the estimated results of Table 1, China's technological innovation level is lower than the advanced countries such as the United States and Germany, but it is close to the developed countries such as France and Canada. It is far higher than the developing countries in India, as well as Spain and Italy, In particular, the proliferation of PV technology in China is relatively low, only 0.594 , not only far below Spain and Italy, but also lower than the advanced technology of the United States and Germany. Therefore, as the largest PV power supplier in the world, its strong development momentum is largely due to the continuous improvement of technological innovation level. 
Table 1. Cross country comparison between technical innovation and imitation level of photovoltaic power

\begin{tabular}{llllll}
\hline & $p$ & \multicolumn{1}{c}{$q$} & \multicolumn{1}{c}{$R^{2}$} & \multicolumn{1}{c}{ RMSE } \\
\hline China & $1.33 \mathrm{E}-06$ & 0.5940 & 511463 & 0.9966 & 1142.665 \\
Germany & $5.04 \mathrm{E}-05$ & 0.7156 & 45501 & 0.9988 & 577.649 \\
U.S.A & $1.36 \mathrm{E}-05$ & 0.4896 & 170492 & 0.9981 & 464.949 \\
France & $8.67 \mathrm{E}-06$ & 0.7694 & 7916 & 0.9933 & 202.357 \\
Britain & $1.23 \mathrm{E}-07$ & 0.9045 & 14921 & 0.9965 & 194.879 \\
Spain & $2.65 \mathrm{E}-09$ & 1.4399 & 5451 & 0.9822 & 321.259 \\
Italy & $1.18 \mathrm{E}-08$ & 1.8869 & 19313 & 0.9982 & 355.197 \\
Australia & $8.40 \mathrm{E}-06$ & 0.7241 & 5799 & 0.9967 & 101.059 \\
Canada & $6.07 \mathrm{E}-06$ & 0.7078 & 3238 & 0.9980 & 38.332 \\
Japan & $6.34 \mathrm{E}-06$ & 0.6403 & 66119 & 0.9956 & 979.186 \\
India & $8.69 \mathrm{E}-07$ & 0.5355 & 218094 & 0.9961 & 140.075 \\
\hline
\end{tabular}

\subsection{Chinese Diffusion prediction of photovoltaic power technology}

Three representative technology diffusion methods, such as standard Bass model (BM), Gompertz model (GM) and Logistic model (LM), are used to predict and analyze the capacity of photovoltaic solar power installed capacity in China. The prediction results are shown in figures 2 and 3.

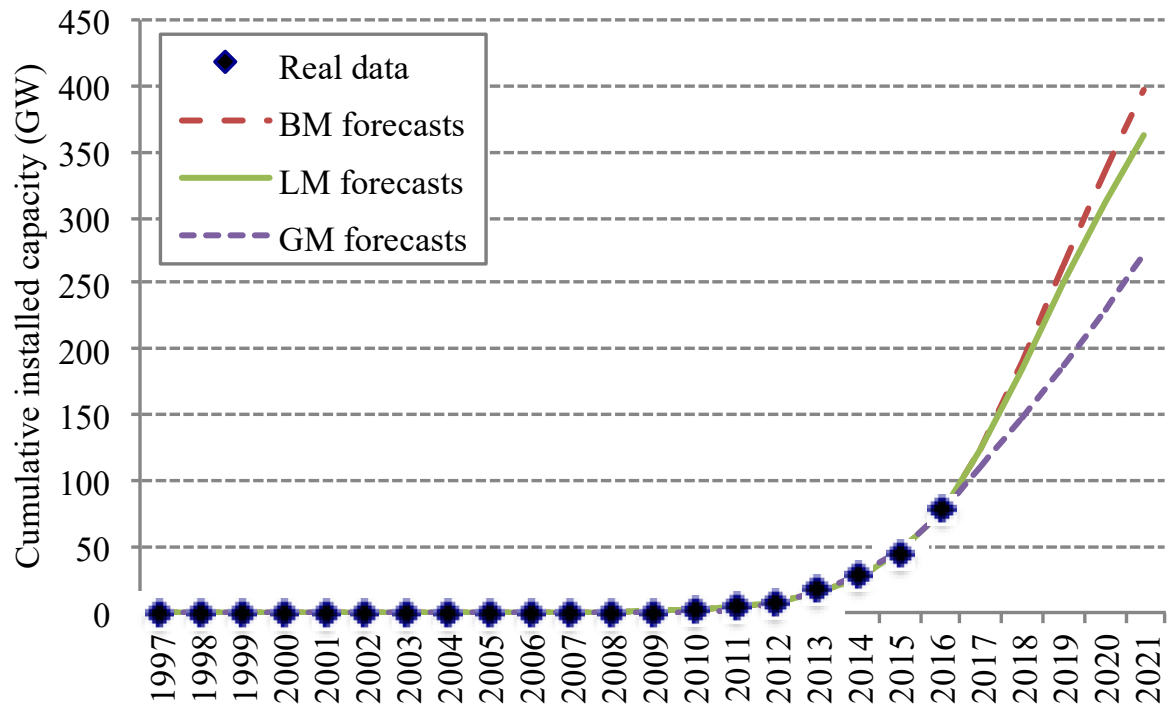

Fig. 2. Cross model predictions of cumulative installed capacity of PV power in China
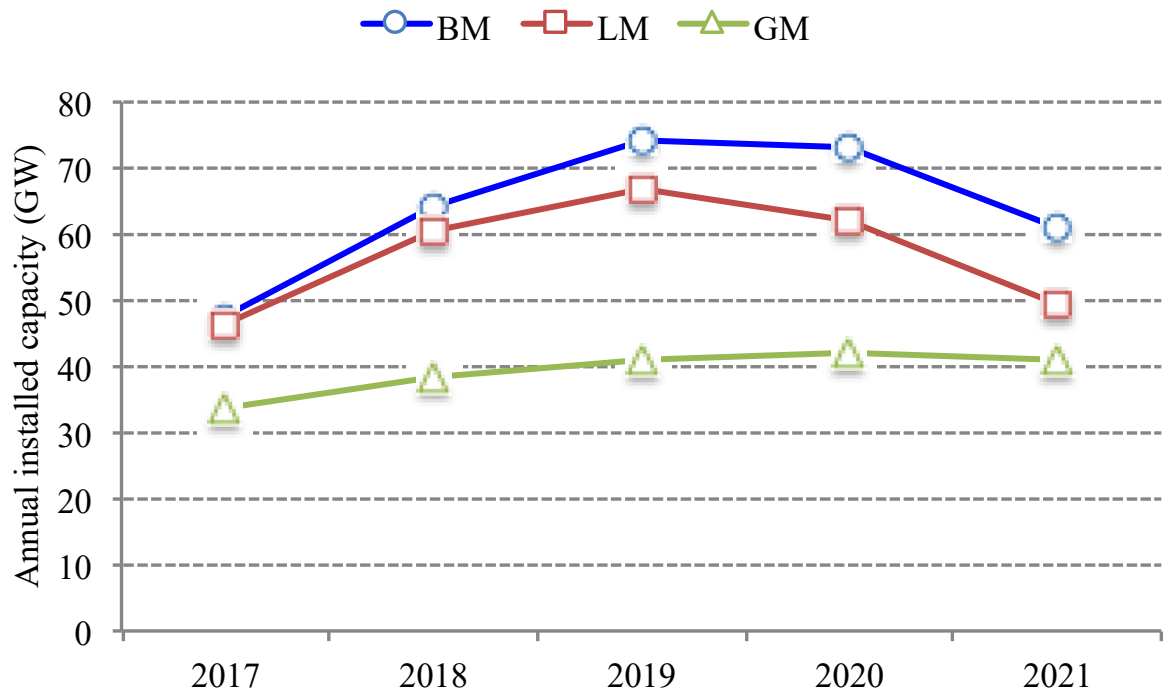

Fig. 3. Cross model predictions of new installed capacity of PV power in China

Figure 2 shows that the prediction result of Bass model is the most optimistic, and the result of
Gompertz model is more conservative, while the prediction value of Logistic model is in the middle. 
This conclusion can also be seen more clearly from the prediction results of new installed capacity (Figure 3). In particular, the conservative GM prediction results, the next 5 years the new installed will show a steady growth, while the BM and LM valuation, diffusion of new installed is showing a significant "hump type". In the past 5 years (2011-2016 years), the cumulative installed capacity of China has increased from 3 billion 550 million watts to 78 billion 70 million watts, an annual increase of $85.54 \%$, and the growth rate of the next 5 years (2016-2021 years) will slow down significantly. The results of the one point three models are consistent. Specifically, Under the estimation of BM, LM and GM, the average annual growth rate of PV installed capacity in China in the next 5 years will decrease to $38.66 \%, 36.15 \%$ and $29.07 \%$. This means that even according to the most optimistic Bass model results, the annual average annual growth rate of photovoltaic power accumulation in China is also lower than $40 \%$ in the next 5 years. By 2021, the total capacity of photovoltaic power installed will be less than 400 billion watts. According to the fitting of historical observation points, Bass model, Gompertz model and Logistic model are applicable to the prediction of photovoltaic power technology diffusion in China, but the prediction accuracy of different models, namely, the average relative percentage error (MAPE), the mean absolute error (MAE) and the median error (MdAPE) are different. The results are shown in Table 2.

Table 2 Comparison of prediction accuracy of each model

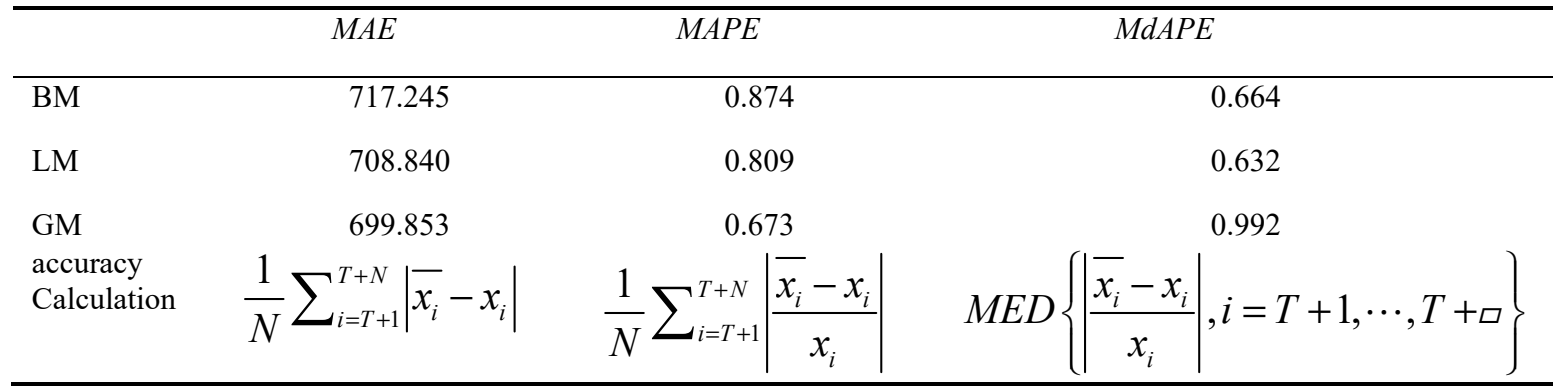

Note: the MED in the table is a median function.

Generally speaking, the fitting degree of three prediction models to our PV power technology diffusion trajectory is better, and the average relative percentage error is less than 1 , The overall prediction accuracy of the Logistic model is the highest, and the standard Bass model is not the best choice. There is a significant difference between the results of the selection of wind power forecast models in China and some European and American countries ([13-16]).

\section{Policy suggestion}

Compared with the advanced countries such as the United States and Germany, the future direction of our efforts should be focused on several aspects: first, to continue to deepen the reform of the power system, to realize the separation of power supply and distribution, and to introduce strong independent power regulators, which is widely regarded as the California, Dezhou and New York regions of the United States. The main reasons for the strong development of photovoltaic power technology are: two, to strengthen the construction of remote distribution network and transmission network, to make full use of the coordination function of the power grid at all levels, to excavate the ability of conventional power peak adjustment and to increase the flexibility of the power grid operation, to maximize the proportion of renewable energy sources such as photovoltaic, and three, to formulate and implement the power purchase method, Do not formulate a detailed and executable classified electricity price sale process, encourage both buyers and buyers to sign long-term contracts and ensure stable and reasonable returns of investors. This is an effective measure to further promote distributed generation mode and reduce "light abandoning rate"; four, continue to carry out long-term protective tariff measures to accelerate green electricity certificates. The establishment of the system of easy system (power quota system), in particular, should not directly cancel the subsidies for photovoltaic power online before the completion of the transaction system and scale implementation. Finally, the role of the government, industry association and mass media at all levels to guide the investment in the research and development of the photovoltaic technology is fully played.

\section{Conclusion}

Using the installed capacity data and Bass model of the most widely developed countries in the world. This paper first makes a cross regional comparison and analysis on the innovation and imitation level of China's photovoltaic power technology diffusion, and then introduces a representative Logistic model and Gompertz model to predict the development of photovoltaic solar technology in the next 5 years in China. Resesrch found: First of all, the level of innovation in photovoltaic power technology is far less than the level of imitation. That is, the market diffusion path of global photovoltaic power technology is dominated by imitation effect. Secondly, despite the late development of photovoltaic power technology in China, the advantages of technology development are more obvious, its innovation level is not only significantly higher than that of Spain, Italy and other 
countries, but also gradually narrowed the gap with the advanced countries such as Germany and the United States. Finally, the results of the cross model forecast show that the peak of the installed capacity of the new photovoltaic power has not yet arrived, the cumulative installed capacity in the next 5 years will still maintain the growth rate of $30 \sim 40 \%$, and the total installed capacity is expected to close to 400 billion watts by 2021.

\section{References}

1. National renewable energy center. The development roadmap of Chinese renewable energy 2050 [R]. The renewable energy development project management office of Zhong Dan.

2. Liu Jianan. Research on optimal strategy of windPV-ES hybrid operation in electricity market[J].Journal of eiectric power science and technology, 2017,32 (1) :11-15.

3. XIE Ting. tudy on coupling power technology for 600 MW supercritical coal-firedgenerating units and distributed photovoltaic energy systems[J]. Journal of eiectric power science and technology, 2015,30(1): 90-104.

4. Duan Hongbo,Zhu Lei,Fan Ying. Analysis of the laws and policies of China's wind energy technology diffusion $[\mathrm{J}]$. Management science, 2013,26(4): 113-120.

5. Shi Cailing,Long Ruyin,Li Huijuan. Research on the diffusion prediction of solar pv technology in China[J]. Industrial and technical economy, 2007, 8: 60-65.

6. Zhou Siqing,Ma Chaoqun,Li Lin. Theory and research on sustainable development of solar photovoltaic industry $[\mathrm{J}]$. The progress and countermeasures of Scientific and technology, 2007, 24(7): 88-90.

7. Yan Huizhen. The application of Gompertz model to population growth prediction $[\mathrm{J}]$. Journal of dalian university of technology, 2015, 34(2): 150152.

8. Bass, F. M.. A new product growth model for consumer durables [J]. Management Science, , 1969,15: 215-227.

9. Chow, G. C. Technological change and the demand for computers [J]. American Economic Review, , 1967, 57(5): 1117-1130.

10. Sundqvist, S., Frank, L. Puumalainen, K The effects of country characteristics, culture similarity and adoption timing on the diffusion of wireless communications $[\mathrm{J}]$. Journal of Business Research, ., 2005.58: 107-110.

11. Mansfield, E. Technical change and the rate of imitation [J]. Econometrica, , 1961,29(4): 741-766.
12. Grilliches, Z., Hybrid corn: an exploration in the economics of technological change [J]. 1957,Econometrica, 25(4): 501-522.

13. Wu, F. S., Chu, W. L.. Diffusion models of mobile telephone [J]. Journal of Business Research, 2010, 63: 497-501.

14. Gruber, H., Verboven, F.. The diffusion of mobile telecommunications services in the European Union [J]. European Economic Review, 2001,45: $577-588$

15. Lee, M., Cho, T. The diffusion of mobile telecommunications service in Korea [J]. Applied Economic Letters, , 2007,14: 477-481.

16. Valle, A. D., Furlan, C.. Forecasting accuracy of wind power technology diffusion models across countries [J]. International Journal of Forecasting, , 2011, 27: 592-601. 